\title{
UAV AND SATELLITE IMAGERY APPLIED TO ALIEN SPECIES MAPPING IN NW SPAIN
}

\author{
Joaquín Martínez-Sánchez ${ }^{1,2}$ *, Luis M. González-de Santos ${ }^{1,2}$, Ana Novo,1 and Higinio González-Jorge ${ }^{2,3}$ \\ ${ }^{1}$ Geotech Group, Mining and Energy Engineering School, University of Vigo, Vigo, Spain - \\ (joaquin.martinez,luismgonzalez,annovo)@uvigo.es \\ ${ }^{2}$ UAS Research Area, Fundación Centro de Innovación Aeroespacial de Galicia, Edificio, Nigrán, Spain.- (joaquin.martinez, \\ luis.gonzalez)@cinae.org \\ ${ }^{3}$ Geotech Group, Aerospace Engineering School, University of Vigo, Ourense, Spain-higiniog@uvigo.es
}

Commission I, ICWG I/II

KEY WORDS: Alien species Mapping, UAS imagery, Satellite remote sensing, Image classification, Photogrametry

\begin{abstract}
:
Image classification stands as an essential tool for automated mapping, that is demanded by agencies and stakeholders dealing with geospatial information. Decreasing costs or UAV-based surveying and open access to high resolution satellite images such as that provided by European Union's Copernicus programme are the basis for multi-temporal landscape analysis and monitoring. Besides that, invasive alien species are considered a risk for biodiversity and their inventory is needed for further control and eradication. In this work, a methodology for semi-automatic detection of invasive alien species through UAV surveying and Sentinel 2 satellite monitoring is presented and particularized for Acacia dealbata Link species in the province of Pontevedra, in NW Spain. We selected a scenario with notable invasion of Acaciae and performed a UAS surveying to outline feasible training areas. Such areas were used as bounds for obtaining a spectral response of the cover from Sentinel 2 images with a level of processing $2 \mathrm{~A}$, that was used for invasive area detection. Sparse detected areas were treated as a seed for a region growing step to obtain the final map of alien species.
\end{abstract}

\section{INTRODUCTION}

Current advances in sensors on-board satellite and UAV platforms are the basis for Earth observation and monitoring with an increasing accuracy and resolution. Remote sensing techniques and open spatial data are essential for gathering information as an input and support for decision-makers.

Richardson and Rejmánek assembled a global list of invasive alien trees and shrubs that describes the distribution of taxa and the spatial representation of 622 species that include, as exceptional, 23 species of the genus Acacia and, among them, the Acacia Dealbata Link species (Richardson and Rejmánek, 2011). There is a focus on this species, because is one of the most widespread alien plant in Europe. In Spain, and specifically in the forested NW regio, it yields an annual spread rate around $0.1 \%$ (Hernández et al., 2014). Existing works comprise the study of biological attributes that are favouring invasion by A. dealbata (Lorenzo et al., 2010) and the production of maps that show how this invasive species is spreading (Martins et al., 2016).

The detection of this invasive species through automated procedures and satellite image processing is a subject with a large presence in the literature, with a focus on public platforms such as Sentinel images (Martimort et al., 2012), ASTER or Landsat (Viana and Aranha, 2010). The possibilities of combining different bands of the electromagnetic spectrum to highlight those elements of the Earth's surface that we are interested in, is an advantage that has been used in innumerable projects (Peña and Ulloa, 2017).

In addition, we can also find works where drones are used for pixel evaluation and image classification. The advantage of these platforms in terms of spatial resolution results in a more detailed cartography, (Mafanya et al., 2017), with a smooth contrast between similar pixels and where the detection is more accurate than with satellite images.
Finally, it is necessary to emphasize that the actual trend in the field has to do with the automation of the detection and mapping. To this end, Machine Learning approaches focused on mutispectral satellite images with several resolutions and bands of the electromagnetic spectrum can be an input for identification and subsequent mapping (Skakun, 2017).

The aim of this work is to develop and test a methodology for mapping Acacia Dealbata Link in the province of Pontevedra, Galicia, a NW Spanish region. The developed methodology is based on UAV-based surveying and the classification of Sentinel 2 L2A imagery.

\section{MATERIALS AND METHODS}

\subsection{Study area}

The species was first introduced in Spain in the first decades of XIX century with ornamental purposes due to its rapid growth and characteristic flowering. The great colonizing capacity of Acacia Dealbata Link has turn its expansion into an environmental issue due to the loss of native species and biodiversity (Lorenzo et al., 2012).

In recent decades, wildfires related deforestation and agriculture abandonment have contributed to the expansion of the species in two main areas: the west and the south of the provinces of Ourense and Pontevedra respectively (Hernández et al., 2014). The density is greater in the western end of the province of Ourense, however, the entire community is dotted with increasingly extensive redoubts of this species. From Portugal, the presence of large areas of Acacia spp. is remarkable and growing, especially in the Baixo Minho region and in the vicinity of the Peneda-Gerês national park.

A first area was used for initial UAV surveying to obtain training data for the classifiers that were afterwards applied to the broader area of work. Figure 1 shows a map of the study area used for

\footnotetext{
* Corresponding author
} 
mapping that comprises the province of Pontevedra, in the Northwest of Spain.

This province comprises an area of $4495 \mathrm{~km}^{2}$, that is suitable to be mapped by means of satellite imagery.

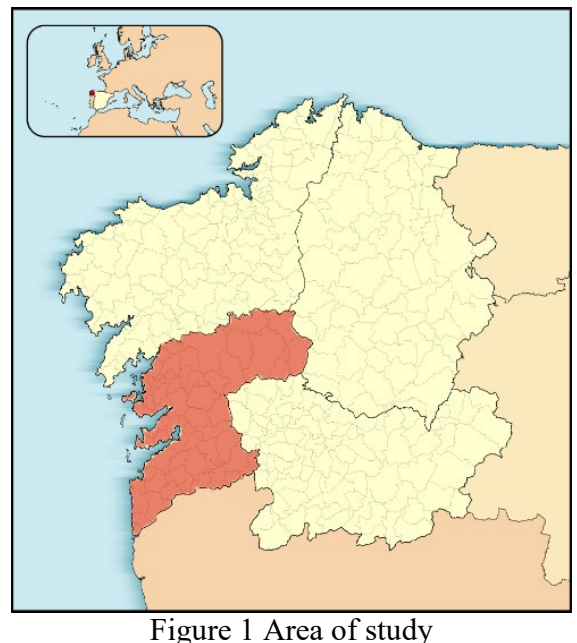

\subsection{Materials}

UAV surveying was performed by a DJI's Phantom 3 Pro. This is a commercial quadcopter that fits the requirements of the project and results very suitable due to the easiness of its work and the automation of the flight.

This quadcopter is equipped with a RGB camera mounted on a gimbal that permits to setup the capture angle and filters the effects of the flight instability on the images. The main attributes of this camera are detailed in Table 1.

\begin{tabular}{|l|c|}
\hline Attribute & Value \\
\hline Sensor & $1 / 2.3^{\prime \prime} \mathrm{CMOS}$ \\
Resolution & $12.4 \mathrm{M}$ \\
Lens & FOV $94^{\circ} 20 \mathrm{~mm}$ \\
Shutter speed & $8 \mathrm{~s}-1 / 8000 \mathrm{~s}$ \\
& \\
\hline
\end{tabular}

Table 1 Phantom 3 Pro camera properties

The flight plan application that was used to setup the requirements in terms of mapping and modelling is the wellknown Pix4DCapture (Pix4D, 2019). This is a free and flexible application that can be used for online and offline mission definition. Based on the definition of the GSD of the survey and the requirements, the application defines the type of survey necessary and establishes the flight altitude and settings most suitable for obtaining the results. In a successive manner, users can set camera angle, image overlap and flight speed. Figure 2 shows the frontend of the used Pix4DCapture application.

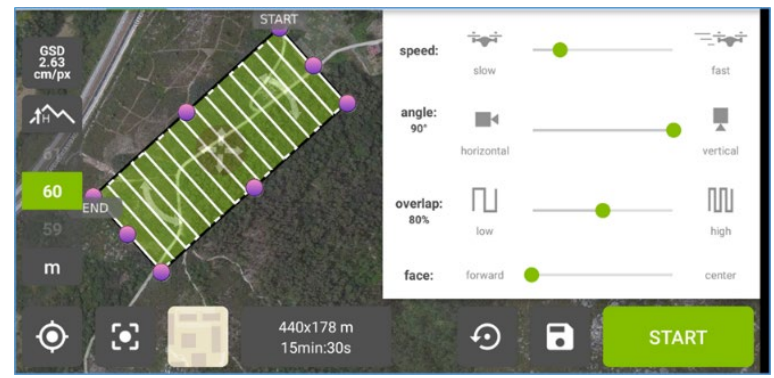

Figure 2 Pix4D App for flight planning
Beyond the high productivity and flexibility posed for UAVs for the capture of information from a specific area, satellite images provide us with images with a global coverage and a suitable revisit time for monitoring forest areas throughout its phenological stages.

In this work we will make use of satellite imagery from the UE Copernicus program. Copernicus aims to collect on a planetary scale environmental data that is intended to be used to mitigate the effects of climate change. This is to improve the quality of life of ecosystems and humanitarian needs in order to more efficiently manage resources in the future and ensure sustainability.

MSI Satellite imagery from the Sentinel 2 mission was used and downloaded from the Copernicus Open Hub. In order to perform a multitemporal classification, L2A Processing Level was selected and multiple season images were downloaded to obtain the classifier (Drusch et al, 2012).

\subsection{Methodology}

\subsubsection{Data acquisition and processing}

In order to obtain a precise footprint of the training areas, a UAV based surveying was performed with the settings detailed in Table 2.

\begin{tabular}{|l|l|}
\hline Parameter & Value \\
\hline Flight Height & $60 \mathrm{~m}(\mathrm{AGL})$ \\
\hline Flight speed & $3 \mathrm{~m} / \mathrm{s}$ \\
\hline Image overlap & $80 \%$ \\
\hline GSD & $2-3 \mathrm{~cm}$ \\
\hline Camera orientation & Nadir \\
\hline
\end{tabular}

Table 2 UAV surveying parameters

Figure 3 shows a collected image of the training survey that may serve as an example of the achieved resolution in the UAV survey

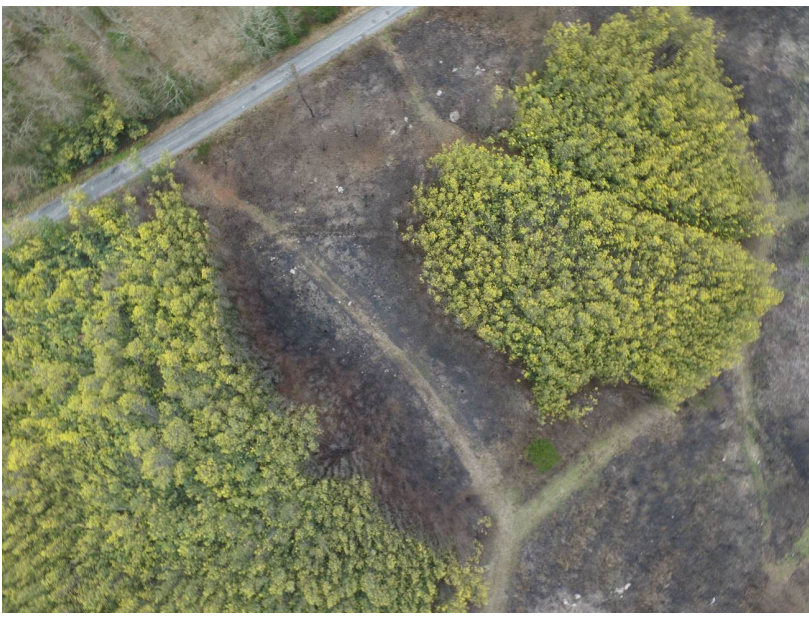

Figure 3 Aerial image collected by the DJI 's Phantom 3 Pro

This aerial images were the input to a photogrammetric bundle adjustment that was processed to obtain both a DEM with the elevation of the training area.

Such DEM is the basis for a orthorectification step that provides an orthomosaic of the area. In our case, a pixel size of $5 \mathrm{~cm}$ was selected, but, given the spatial resolution of the satellite imagery, this setting is more focused on documentation purposes than training requirements. Figure 4 shows the resulting orthomosaic of one of the training areas in the work.

The resulting orthomosaic is suitable for delineation of the training area which is depicted in Figure 5. 


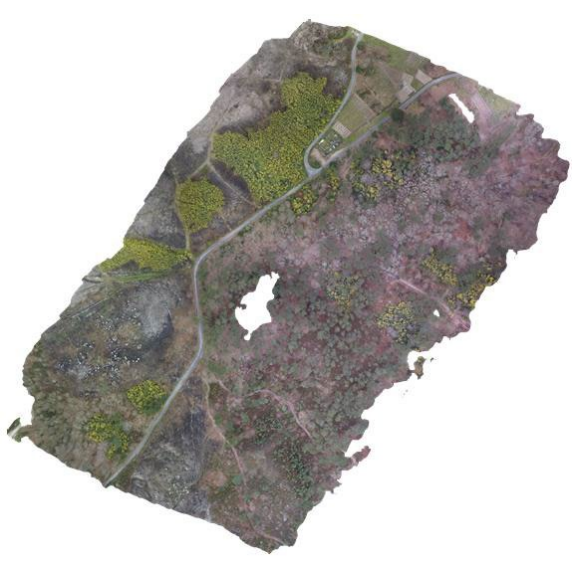

Figure 4 Resulting orthomosaic of the photogrammetric survey based on UAV

In order to obtain the satellite imagery, a script has been developed for the automatic download of Sentinel2 images from the province of Pontevedra. The script is prepared to run in an unattended process and, perform the query, download the images, if new images are available, and generate the mosaic of the province of Pontevedra.

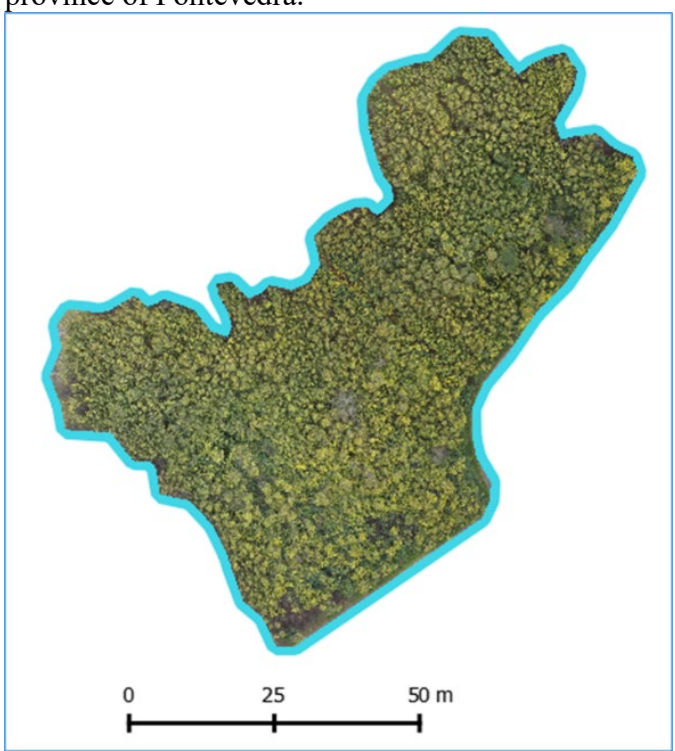

Figure 5 Training area delineation. This footprint is to be used as an overlay of the satellite images.

The access to the Sentinel Hub repository is made through the REST service of the hub, that provides an API to access the database through a query. This query has a series of parameters that allow obtaining the most appropriate images for the specific application. In our case we will access the images of processing level L2A (estimation of the reflectance in the lower part of the atmosphere).

As a second stage, an orthomosaic of the province is computed. Depending on the satellite orbit, the province of Pontevedra will appear in a number of images between 2 and 4 . From these images a mosaic of all of them is generated to have a single image showing the area of the province in all its extension. Figure 4 shows an example of one of the generated orthomosaics of the province.

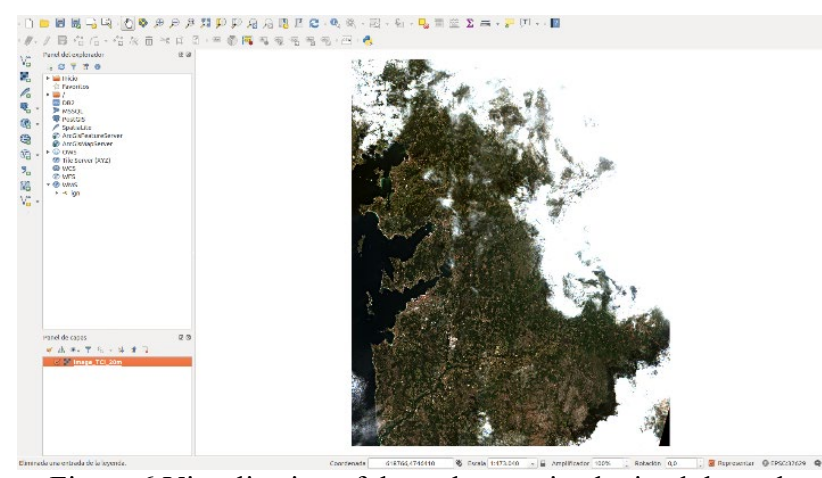

Figure 6 Visualization of the orthomosaic obtained through Sentinel L2A images processing

\subsubsection{Data analysis and classification}

In the second step of the methodology, previous footprints are used as Regions of Interes (ROI) for the classification of the alien species. This classification was carried out using the Semiautomatic Classification Plugin in QGis software (Congedo, 2016).

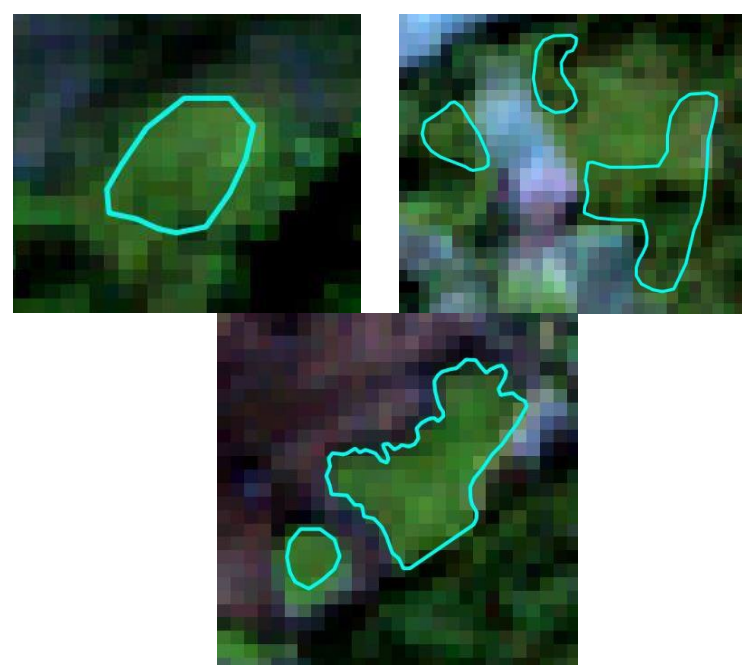

Figure 7 ROIs used for training in the classification. The ROIs are overlaid on a composition of Sentinel 2 L2A bands 3-2-1.

The classification was performed making use of the spectral information of the satellite images. Some examples of the spectral detectors based on the pixel information in L2A images is depicted in Figure 8. The more pixels considered to obtain this estimation, the more information about the spectral detector to be used.

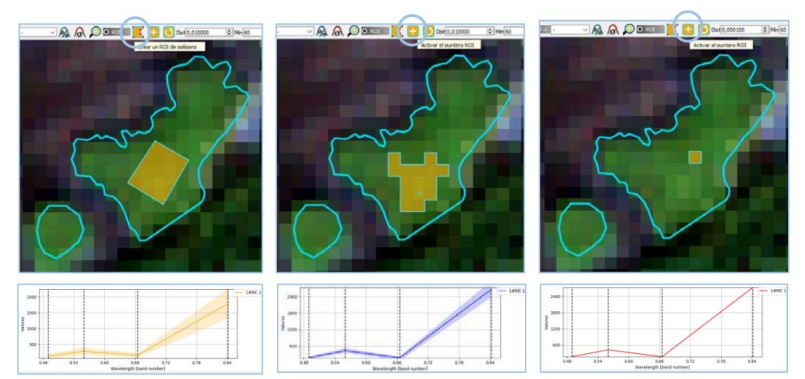

Figure 8 Spectral detector obtained using SCP with different selection parameters. Depending on the number of pixels in the considered region, we obtain an estimator of the spectral signature of the species. 
The training areas obtained with the photogrammetric survey were used as bounds for obtaining a spectral response of the cover from Sentinel 2 images with a level of processing $2 \mathrm{~A}$. This spectral response serves as an estimator of the spectral signature of the species, and it was used for invasive area detection.

Taking into account the distance between a pixel and the spectral estimator, a pixel is classified as alien species.

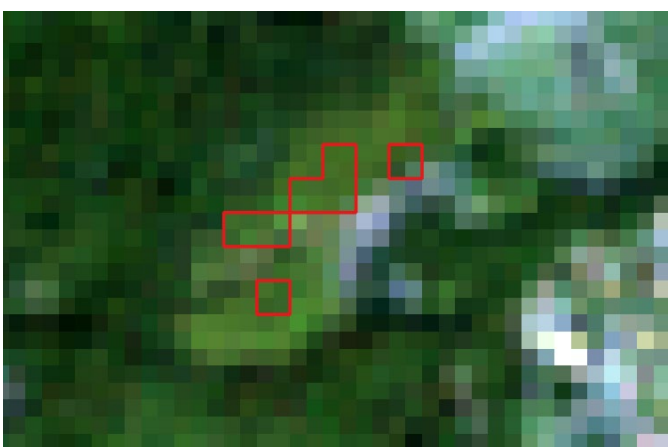

Figure 9 Result of the detection based on the distance between a pixel and the spectral detector obtained with SCP plugin.

\subsubsection{Cartography and mapping}

The spectral classifier defined from the pixels of the training zones does not generalize the results correctly. As a result, there are a large number of pixels belonging to areas of acacias sp. that are not classified correctly.

However, a large number of pixels classified as acacia are correct and the number of false positives in the classification is very low. Therefore, we can consider the classifier as an Acacia $s p$. detector, in which at least one pixel of the blobs of this species is detected correctly.
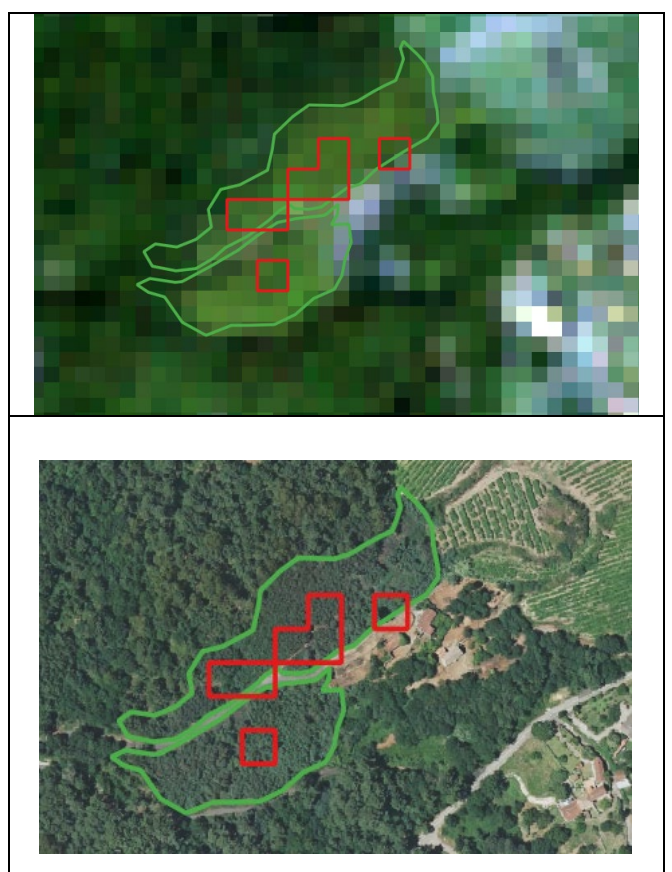

Figure 10 Detected pixels in a area of Acacia overlaid on a Sentinel 2 image (up) and an official orthophoto (bottom)

Finally, to obtain the map of acacias in the study area, sparse detected areas were treated as a seed for a region growing step to obtain the final map of alien species.

\section{RESULTS}

Figure 11 shows the results of the detection of acacia zones based on the spectral distance between the pixels of the image and the estimator obtained from the training areas.

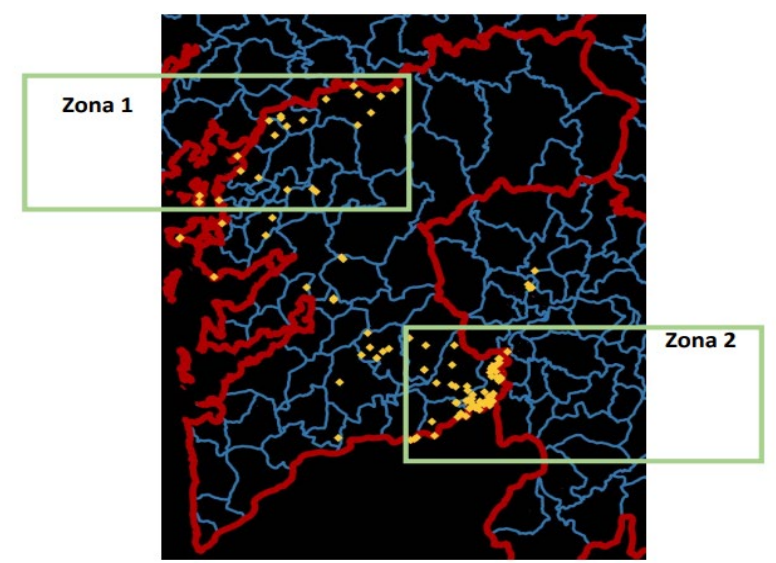

Figure 11 Results of detection based on the spectral distance of the estimator

As aforementioned in the previous section, the defined estimator can not be considered as a classifier, since there are a large number of pixels belonging to the invasive species that have not been classified correctly. However, a large percentage of the classified pixels corresponds to correct pixels and, therefore, the estimator has been considered as a detector of blobs containing the species considered.

Given the large extension of area under study in this study, two sampling areas have been defined to assess this detection. The precision of the detection and the number of false positives have been verified from the positive pixels obtained by the classifier in those sampling areas, that are depicted in Figure 11.

From this selection of pixels, it has been found that the detection accuracy has been $86 \%$ and, consequently, the percentage of false positives is $14 \%$.

Finally, from the detected areas, the estimated invasion map has been compiled, which is presented in figure 12. In this map, the administrative divisions in which the condition is observed are highlighted.

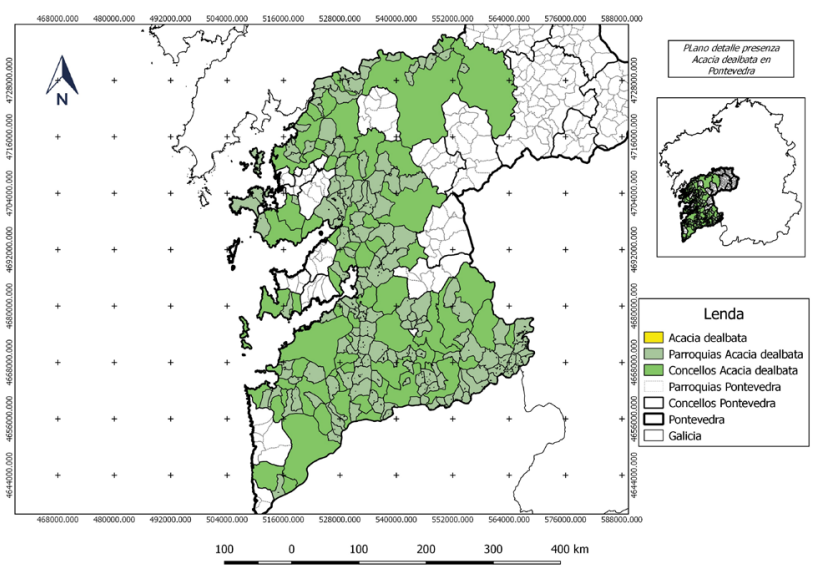

Figure 12 Acacia sp. map obtained. The Municipalities with positive detections of alien species are highlighted in green. 


\section{CONCLUSIONS}

The combination of satellite platforms and UAVs allows us to take advantage of the synergy between the global coverage of the former and the spatial resolution of the latter.

The high productivity obtained from these Systems of Systems, implies that they can be considered as an alternative to other methods of land observation of greater costs.

In this work, a detector has been developed that is focused on the classification of invasive forest species with the idea of generating a map of their evolution.

The revisit time of the satellites allows us to have a large number of samples of images in different phenological stages of the species, and therefore, adapt the classifier to the time of year in which it is being measured.

On the other hand, the absence of sufficiently large areas of uniform extensions of the species, makes the resolution of the satellite images a handicap to achieve a correct classification of all the pixels corresponding to the invasive species.

However, the classifier has given good results as a detector of the areas in which we can find the invasive species, and we have used this feature to perform a growth of the detected regions to generate a map of affected areas.

This mapping process still requires a lot of manual work and therefore, future lines of work should be aimed at achieving an automation of the classifiers.

\section{ACKNOWLEDGEMENTS}

Authors would like to acknowledge Deputación de Pontevedra for the grant 17/410.1720.789.02 and Jose Antonio Ruiz for his invaluable collaboration in this work.

\section{REFERENCES}

Congedo, L., 2016. Semi-Automatic Classification Plugin Documentation. doi.org/10.13140/RG.2.2.29474.02242/1

Drusch, M., Del Bello, U., Carlier, S., Colin, O., Fernandez, V., Gascon, F., ... \& Meygret, A., 2012. Sentinel-2: ESA's optical high-resolution mission for GMES operational services. Remote sensing of Environment, 120, 25-36.

Hernández, L., Martínez-Fernández, J., Cañellas, I., Cueva, A. V., 2014. Assessing spatio-temporal rates, patterns and determinants of biological invasions in forest ecosystems. The case of Acacia species in NW Spain. Forest Ecology and Management, 329, 206-213.

Lorenzo, P., González, L., Reigosa, M. J. ,2010. The genus Acacia as invader: the characteristic case of Acacia dealbata Link in Europe. Annals of Forest Science, 67(1), 101.

Lorenzo, P., Pazos-Malvido, E., Rubido-Bará, M., Reigosa, M. J., González, L., 2012. Invasion by the leguminous tree Acacia dealbata link (Mimosaceae) reduces the native understorey plant species in different communities. Australian Journal of Botany, 60, 669-675.

Mafanya, M., Tsele, P., Botai, J., Manyama, P., Swart, B., Monate, T., 2017. Evaluating pixel and object based image classification techniques for mapping plant invasions from UAV derived aerial imagery: Harrisia pomanensis as a case study. ISPRS Journal of Photogrammetry and Remote Sensing, 129, 1 11.
Martins, F., Alegria, C., Artur, G., 2016. Mapping invasive alien Acacia dealbata link using ASTER multispectral imagery: a case study in central-eastern of Portugal. Forest systems, 25, 13.

Martimort, P., Fernandez, V., Kirschner, V., Isola, C., Meygret, A. 2012. Sentinel-2 MultiSpectral imager (MSI) and calibration/validation. Geoscience and Remote Sensing Symposium (IGARSS), 2012 IEEE International, (págs. 69997002).

Peña, M. A., Ulloa, J. ,2017. Mapeo de la recuperación de la vegetación quemada mediante la clasificación de índices espectrales pre-y post-incendio. Revista de Teledetección, (págs. 37-48).

Pix4D SA, 2019. https://www.pix4d.com/product/pix4dcapture, Accessed on 12 Feb., 2019

Richardson, D. M., Rejmánek, M. ,2011. Trees and shrubs as invasive alien species-a global review. Diversity and distributions, 17(5), 788-809.

Skakun, S. a.-C., 2017. Automatic sub-pixel co-registration of Landsat-8 Operational Land Imager and Sentinel-2A MultiSpectral Instrument images using phase correlation and machine learning based mapping. International Journal of Digital Earth.

Viana, H., Aranha, J., 2010. Mapping invasive species (Acacia dealbata Link) using ASTER/TERRA and LANDSAT 7 ETM+ imagery. Forest Landscapes and Global Change-New Frontiers in Management, Conservation and Restoration. Proceedings of the IUFRO Landscape Ecology Working Group International Conference. 